\title{
Cell Membrane Coated-Biomimetic Nanoplatforms Toward Cancer Theranostics
}

\author{
Tingting $\mathrm{Li}^{1,2}$, Xiang Qin ${ }^{1,2}$, Yichao $L i^{1}$, Xue Shen ${ }^{3}$, Shun $\mathrm{Li}^{1,2}$, Hong Yang ${ }^{1,2}$, \\ Chunhui Wu ${ }^{1,2}$, Chuan Zheng ${ }^{4}$, Jie Zhu ${ }^{4}$, Fengming You ${ }^{4}$ and Yiyao Liu ${ }^{1,4 *}$ \\ ${ }^{1}$ Department of Biophysics, School of Life Sciences and Technology, University of Electronic Science and Technology \\ of China, Chengdu, China, ${ }^{2}$ Center for Information in Biology, University of Electronic Science and Technology of China, \\ Chengdu, China, ${ }^{3}$ Sichuan Industrial Institute of Antibiotics, Chengdu University, Chengdu, China, ${ }^{4}$ Department of Cancer \\ Research, Hospital of Chengdu University of Traditional Chinese Medicine, Chengdu, China
}

OPEN ACCESS

Edited by:

Valerio Voliani,

Italian Institute of Technology (IIT), Italy

Reviewed by:

Nicolò Mauro,

University of Palermo, Italy

Aether Junqing Wang,

Harvard Medical School,

United States

Dan Shao,

South China University of Technology,

China

*Correspondence:

Yiyao Liu

liuyiyao@uestc.edu.cn

Specialty section:

This article was submitted to

Nanobiotechnology,

a section of the journal

Frontiers in Bioengineering and

Biotechnology

Received: 13 February 2020

Accepted: 03 April 2020

Published: 29 April 2020

Citation:

Li T, Qin X, Li Y, Shen X, Li S,

Yang $H$, Wu C, Zheng C, Zhu J, You F and Liu Y (2020) Cell Membrane Coated-Biomimetic Nanoplatforms

Toward Cancer Theranostics.

Front. Bioeng. Biotechnol. 8:371.

doi: 10.3389/fbioe.2020.00371
Research of nanotechnology for cancer therapy and diagnosis extends beyond drug delivery into the targeted site or surveillance the distribution of nanodrugs in vivo or distinction tumor tissue from normal tissue. To satisfy the clinic needs, nanotheranostic platform should hide the surveillance by immune system and the sequestration by filtration organs (i.e., liver and spleen). Use of biologically derived cellular components in the fabrication of nanoparticles can hide these barriers. In this review, we update the recent progress on cell membrane-coated nanoparticles for cancer theranostics. We hope this review paper can inspire further innovations in biomimetic nanomedicine.

Keywords: cancer, theranostic, cell membrane, biomimetic, nanocomplex

\section{INTRODUCTION}

Cancer is known to be the most aggressive malignancy to humans, and definitely the major cause of death worldwide (Roma-Rodrigues et al., 2019). In the fight against cancer, half of the battle is won based on its early detection (Phillips et al., 2014; Ye et al., 2014). As we know, new treatments such as phototherapy and immunotherapy have received considerable attention due to their obvious advantages than the conventional therapies. Phototherapy including photothermal therapy (PTT) and photodynamic therapy (PDT), they rely on the phototherapeutic agents in cooperation with laser irradiation to selectively kill cancer cells, while ignoring the healthy cells in the dark (Liu X. et al., 2018; Li W. et al., 2019). However, monotherapies have limited efficacy. Consequently, multiple approaches have been provided to be a promising route for cancer therapy (Min et al., 2019; Yue et al., 2019).

Theranostics based on nanotechnology strategies is a new form of cancer treatment, they can integrate the conventional or the new therapeutic modalities and diagnostic functions such as magnetic resonance (MR) imaging, photoacoustic (PA) imaging, positron emission tomography (PET) imaging or fluorescence imaging etc. into one single carrier and assist in the management of cancer (Li et al., 2016; Li T. et al., 2019). Theranostics showed a number of advantages such as improved diagnosis, tumor specific delivery of drugs, reduced damage to healthy tissue.

In the last few decades, theranostic nanoplatforms gained great progress in basic research and produced a large number of excellent publications (Yang H. et al., 2018; 


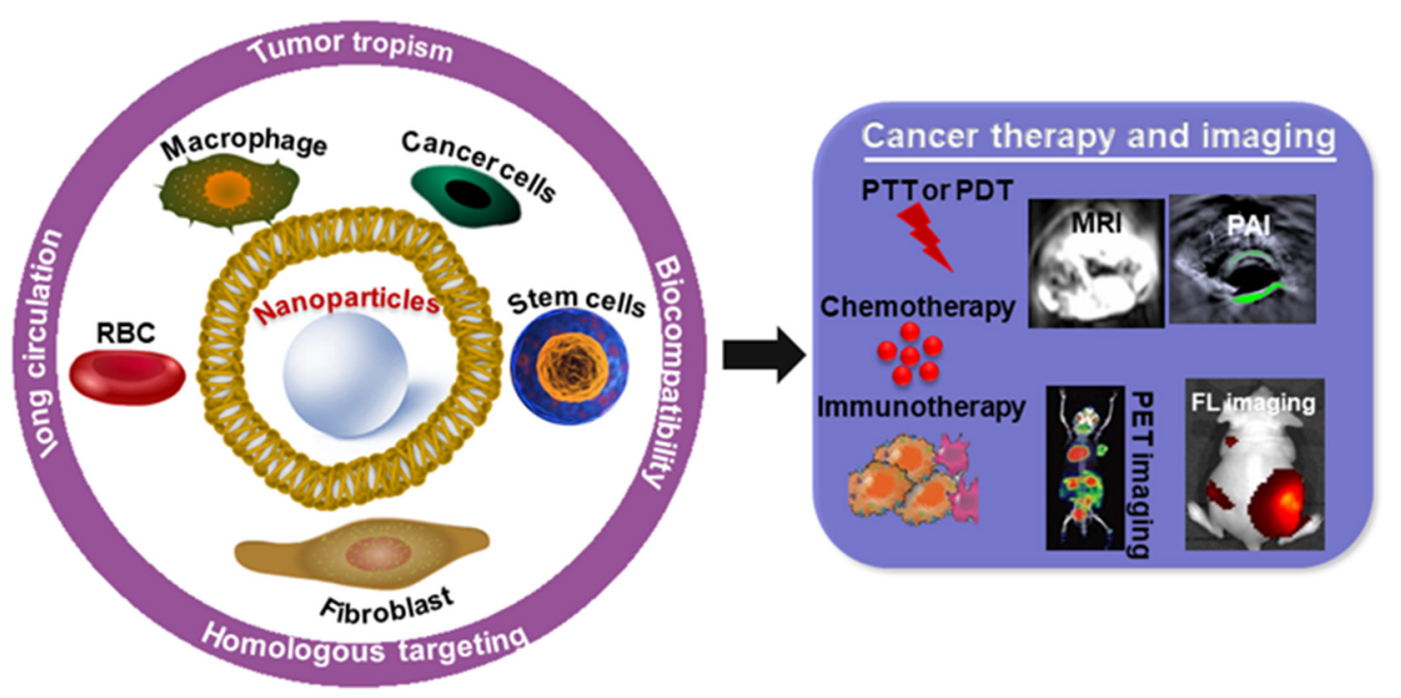

FIGURE 1 | The application of cell membrane-coated nanoparticles for cancer therapy and imaging.

Shen et al., 2019). Scientists developed numerous theranostic nanosystems based on organic nanoparticles, inorganic nanoparticles, micelles, dendrimer, and got good effect in cell studies and animal studies (Ray et al., 2018; Yang J. et al., 2018; Shao et al., 2019; Xu et al., 2019; Bort et al., 2020). These theranostic nanoplatforms were modified with polymer, antibody, peptide or other functional molecules to obtain profuse biological functions, such as targeting, long circulation, biocompatibility, and immune escaping (Yang et al., 2017; Liu S. et al., 2019; Xie X. et al., 2019; Wu et al., 2020).

But the application of theranostic nanoplatforms in the clinical trials have been disappointing. Two vital problems need to be solved urgently, the first one is the biosafety of theranostic platforms should be systemically evaluated. The second one, which our final goal, is getting the expected effect in the clinic (Choi et al., 2012; Liu Y. et al., 2018; Vankayala and Hwang, 2018; Jing et al., 2019). As a consequence, we should develop theranostic nanosystems that closely mimic the biological composition of our bodies and make the efficiency of theranostic nanoplatforms maximally.

Cell membrane coated biomimetic nanoplatforms are often semi-biological (or semi-artificial) which take advantages of their inherited property, such as biointerfacing, self-identification and signal transduction can escape from biological barriers such as immune clearance, opsonization, and negotiation with vascular system (Evangelopoulos and Tasciotti, 2017; Chen et al., 2019; Jin et al., 2019; Madamsetty et al., 2019; Yan et al., 2019; Ma et al., 2020a). These theranostic nanoplatforms have the potential to play an important role in cancer diagnosis and treatment (Bose et al., 2018a; Li Z. et al., 2018; Meng et al., 2018; Sung et al., 2019; Ye S. et al., 2019). This review article will introduce the recent efforts on the rational design of cell membrane-based biomimetic nanosystem for cancer diagnosis and treatment, we highlight the strategies of engineering and application in Figure $\mathbf{1}$.

\section{DEVELOPMENT OF CELL MEMBRANE-COATED BIOMIMETIC THERANOSTIC NANOPLATFORM}

Hu et al. (2011) first reported that they used a top-down method to synthesize erythrocyte membrane camouflaged nanoparticles for long-circulating cargo delivery. Since that, cell membrane, not just erythrocyte membrane but cancer cell membrane, stem cell membrane, platelet membrane, endothelial cell membrane, etc. were used for coating materials of nanoparticles (Kroll et al., 2017; Narain et al., 2017; Pasto et al., 2019; Zhou et al., 2019). Owning the advantages of the native functionalities originating from cell membrane including reserved antigens and cell membrane structure, biomimetic nanoparticles can acquire special functions, such as ligand recognition and targeting, long blood circulation, and immune escaping (Chen Z. et al., 2016; Feng et al., 2019; Meng et al., 2019). In a valuable review paper, the authors discussed in detail the advantages of different cell membrane camouflaged nanoparticles (Chen Z. et al., 2016). In this paper, we briefly summarized the recent progress in the development of biomimetic cell membrane camouflaged nanocomplex for cancer theranostic. We also demonstrated the highlight in the recent research about biomimetic cell membrane camouflaged nanocomplex on cancer theranostic in the following sections.

\section{RED BLOOD CELLS MEMBRANE CAMOUFLAGED THERANOSTIC NANOCOMPLEX}

Red blood cells (RBCs) are the primary transport of oxygen through the blood in body, they can live up to 120 days in humans, and are nature's long circulating carriers 
(Hsieh et al., 2015; Fang et al., 2018; Liu J.M. et al., 2018). It is reported that RBCs are the ideal membrane modification materials because of the abundant proteins, glycans, and receptors on the RBCs membrane surfaces which can bypass the immune system attack (Rao et al., 2017; Ren et al., 2017; $\mathrm{Su}$ et al., 2017). For example, CD47 (integrin-associated protein) is a self- marker of RBCs which can interface with its corresponding receptor, prevent the clearing from the bloodstream by macrophages (Oldenborg et al., 2000; Xie J. et al., 2019). The intact RBCs membrane could directly modify on the surface of nanoparticles without any complex process, and the final nanoparticle still inherits the functions of RBCs (Jiang et al., 2017; Lian et al., 2019; Yang et al., 2019). In one instance, Li et al. used $\mathrm{Ag}_{2} \mathrm{~S}$ quantum dots (QD), a good fluorescence imaging agent with ideal photothermal and photodynamic therapeutic effects under laser irradiation as a sonosensitizer. Pluronic F-127-modified $\mathrm{Ag}_{2} \mathrm{~S}$ QDs were wrapped in $\mathrm{RBC}$ vesicles for enzyme-augmented sonodynamic therapy (SDT). RBC membranes coating in this system could prolong the circulation time of the probe, and catalyzed endogenous $\mathrm{H}_{2} \mathrm{O}_{2}$ by the catalase in RBCs to ameliorate tumor hypoxia. Besides, Ultrasound (US) could also promote tumor blood flow, relieve the hypoxic condition, and enhance the SDT effect of the probe. This study provide a promising strategy for the future design of a multifunctional theranostic nanoplatform (Li et al., 2020). Wang and co-workers designed $\mathrm{RBC}$ based probe (RBCp) for NIR-II fluorescence bioimaging-guided tumor surgery and light-triggered $\mathrm{O}_{2}$ release to enhance PDT efficiency. In vivo study showed that RBCp could provide efficient tumor targeting and laser-responsive $\mathrm{O}_{2}$ release to enhance the PDT efficiency of popliteal lymph node metastasis under the guidance of NIR II fluorescence bioimaging (Figure 2; Wang et al., 2019).

\section{CANCER CELL MEMBRANE CAMOUFLAGED THERANOSTIC NANOCOMPLEX}

Inspired by the reality that nanotheranostic nanoplatform should have good biocompatibility and the ability of homologous targeting, cancer cell membrane coated nanotheranostic nanoplatform have been recently getting more and more attention (Li S.Y. et al., 2018; Zhang N. et al., 2018; Zhang W. et al., 2019; Kumar et al., 2019). In particular, cancer cells are robust and easy to multiply culture in vitro for mass membrane collection, cancer cell membrane expressing "markers of self" and "self-recognition molecules" can be removed from cancer cells and coated on nanoparticles, demonstrating homologous targeting and immune escape ability (Bose et al., 2018b; Shao et al., 2018; Cai et al., 2019; Harris et al., 2019; Liu C. et al., 2019; Nie et al., 2019; Zhang D. et al., 2019). Wang and coauthors designed HeLa cell membrane coated nanocomposites for Fluorescence/MR dual-modal imaging guided PDT. These HeLa membrane coated nanocomposites (denoted as mGZNs) showed enhanced in vivo anti-tumor targeting efficiency of $80.6 \%$ for HeLa cells, providing new strategies to develop nanocomposites for visualized cancer theranostics (Wang et al., 2020). Zhu and coworkers designed a magnetic iron

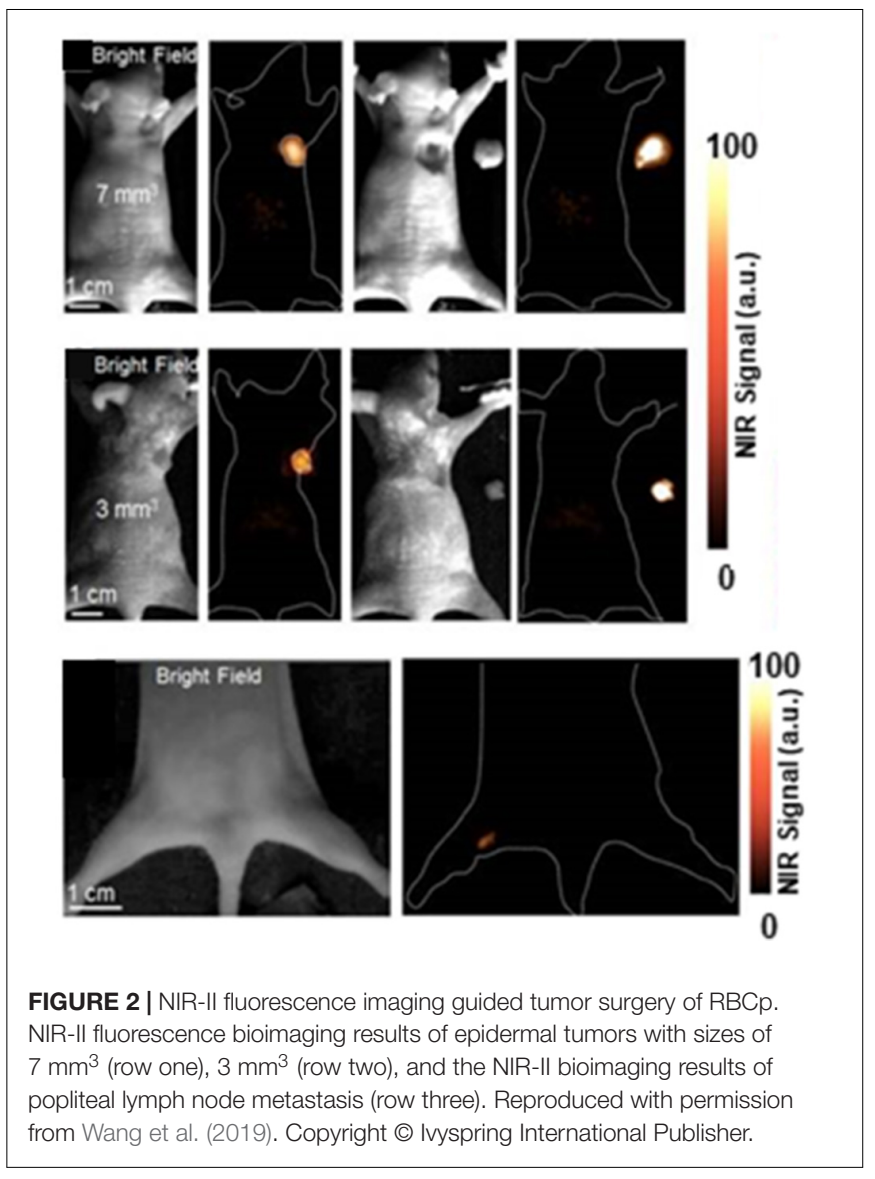

oxide based nanosystem coated with different types of cracked cancer cell membranes (CCCM). This nanocomplex showed the excellent self-recognition internalization by the source cancer cell lines in vitro and in vivo. As shown in Figure $\mathbf{3 A}$, cellular internalization of UM-SCC-7, and HeLa cell membrane coated MNP@DOX@NPs (termed as MNP@DOX@UM-SCC-7 and MNP@DOX@HeLa, respectively) was studied upon $3 \mathrm{~h}$ coincubation with four cell lines including UM-SCC-7, HeLa, HepG2, and COS7 cells. An amazing outcome was found that the fluorescence intensity originating from two CCCM coated nanoparticles was far superior in the corresponding source cells over those in heterotypic cells. To conform the in vivo tumor self-targeting ability toward homologous tumors, the authors intravenously injected mice bearing UM-SCC-7 tumor on the right hind limb with MNP@DOX@CCCM NPs prepared with different cell membranes. As shown in Figure 3B, In the group injected with MNP@DOX@UM-SCC-7 showed more intratumor fluorescence intensity than other groups (Zhu et al., 2016).

\section{STEM CELL MEMBRANE CAMOUFLAGED THERANOSTIC NANOCOMPLEX}

Stem cell membrane is another natural biomimetic membrane coating that have been used for cancer theranostics (Ma et al., 2019; Shin et al., 2019; Zhao et al., 2019). Stem cell membrane 


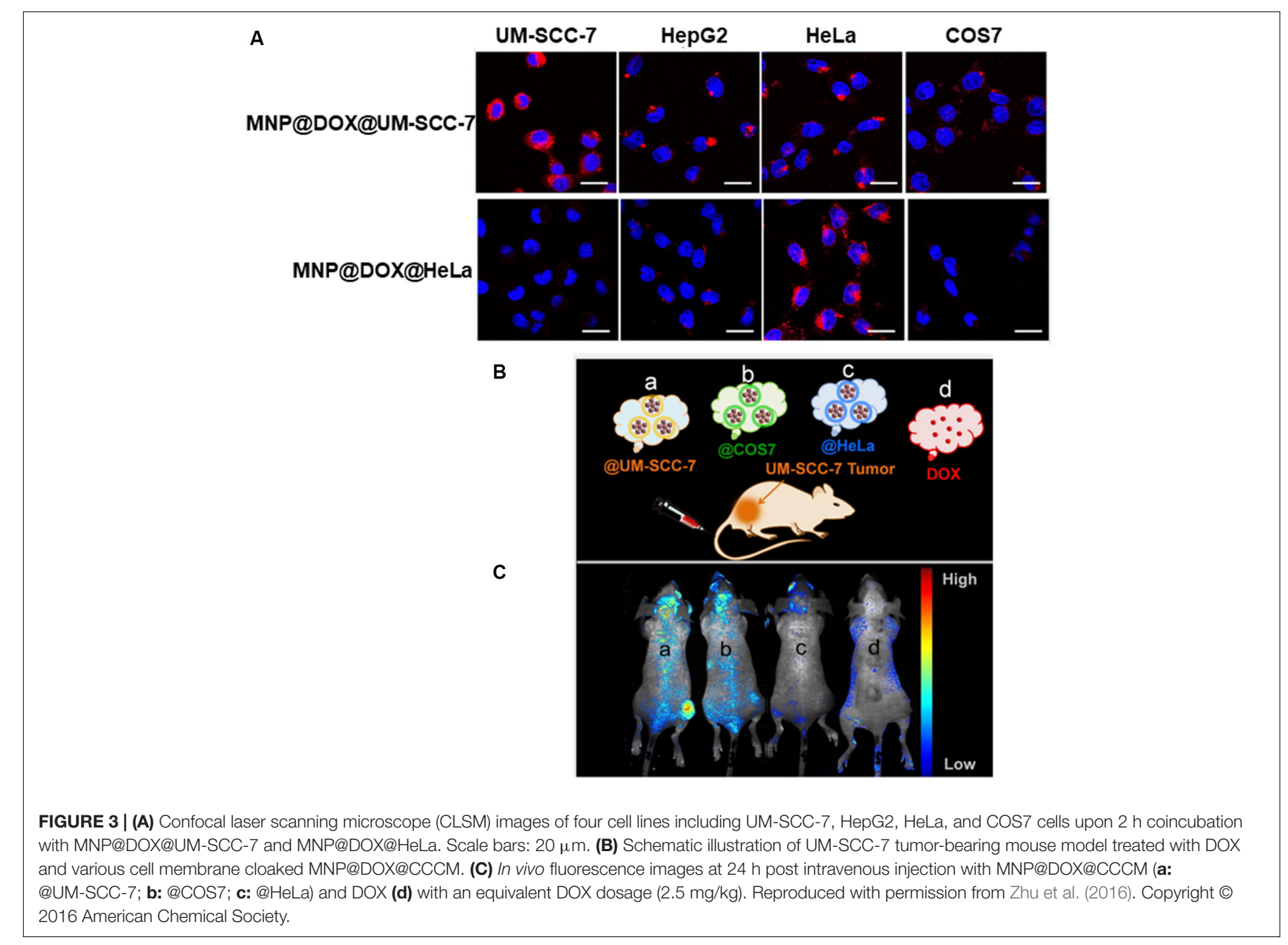

with its inherent tumortropism coating onto nanoparticles has enabled the fabrication of nanocarriers with similar targeting functionality (Letko Khait et al., 2019; Wu et al., 2019). In an example of umbilical cord-derived mesenchymal stem cell coated polymeric nanoplatform, poly(lactic-co-glycolic acid) (PLGA) nanoparticle loaded with Doxorubicin (NP-Dox) were coated with cord-derived mesenchymal stem cell membrane for tumortargeted delivery of chemotherapy. The coating membrane significantly enhanced the cellular uptake efficiency of PLGA nanoparticles and the tumor cell killing efficacy of PLGAencapsulated doxorubicin (Yang N. et al., 2018). In another study, bone marrow derived mesenchymal stem cell membrane was coated on gelatin nanogels (termed as SCMGs) for tumortargeted drug delivery. SCMGs showed high cancer cellular uptake of DOX compared with gelatin-DOX and free DOX. To monitor the in vivo distribution of nanogels, a nearinfrared fluorescent dye, Cyanine7 (Cy7) was loaded into both SCMGs and bare gelatin nanogels. After intravenous injection of different nanogel formulations in tumor bearing mice, the average fluorescence signal in the SCMGs treated mice was notably higher than that obtained in the group treated with bare gelatin (Gao et al., 2016).

\section{CANCER-ASSOCIATED FIBROBLAST MEMBRANE CAMOUFLAGED THERANOSTIC NANOCOMPLEX}

Cancer associated fibroblast membrane has recently obtained more and more attention as membrane coating materials. As reported, cancer-associated fibroblasts are recognized as a key obstacle to cancer treatment (Chen B. et al., 2016). On the one hand, they construct a protecting physical barrier to impede tumor cells uptake of antitumor drugs. One the other hand, they secrete abundant growth factors and cytokines to activate correlative signaling pathways for promoting tumor angiogenesis, progression, initiation, metastasis, and resistance. Studies have shown that used cancer-associated fibroblast membrane coated nanoparticles to deliver therapeutic agents could target and kill cancer-associated fibroblasts, and depleted tumor-stroma biological interactions and in turn led to enhanced therapy (Ji et al., 2015, 2016; Li L. et al., 2018; Kovacs et al., 2020). Li et al. developed semiconducting polymer nanoparticles (SPNs) coated with activated fibroblast membranes (denoted as AF-SPN) for enhanced multimodal cancer phototheranostics. In this study, uncoated SPN (uSPN) nanoparticles and the cancer 


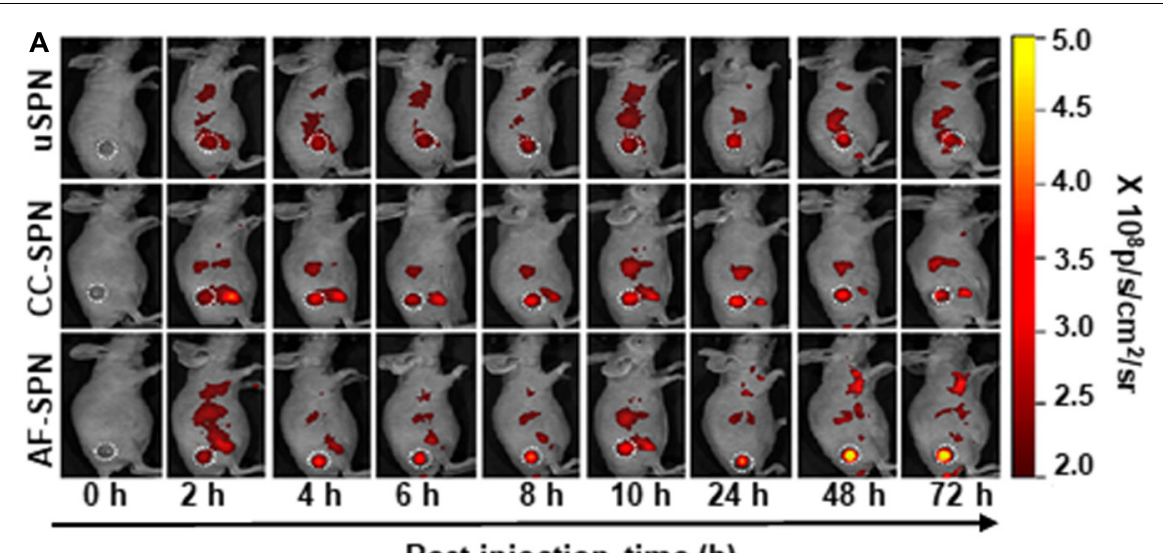

Post-injection time (h)

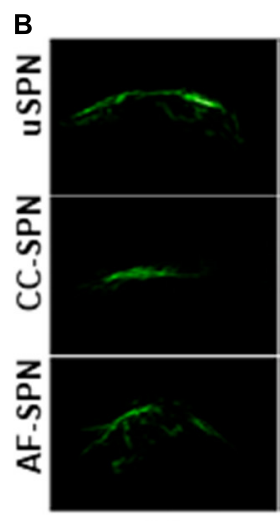

$\mathrm{Oh}$

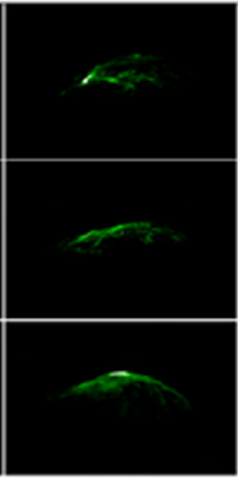

$24 \mathrm{~h}$

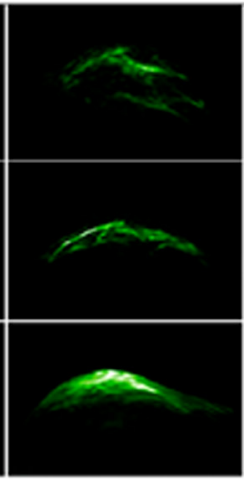

$48 \mathrm{~h}$
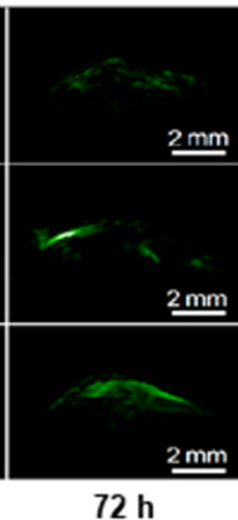

$\operatorname{Max}$

Min

FIGURE 4 | In vivo fluorescence and PA imaging of tumors in living mice after treatment with different complexes. (A) In vivo NIR fluorescence images of 4T1 tumor-bearing mice at different time points after systemic administration of uSPN, CC-SPN, and AF-SPN via tail vein injection. The tumor was on the right side of the back, as indicated by the white dashed circles. (B) Representative PA maximum imaging projection of 4T1 tumors in living mice at 0 , 24 , 48, and $72 \mathrm{~h}$ after systemic administration of different complexes via tail vein injection. Reproduced with permission from Li J. et al. (2018). Copyright @ 2018 American Chemical Society.

cell membrane coated SPN (CCSPN) nanoparticles were as control group. To demonstrate the photodiagnostic potential of nanoparticles in vivo, different nanoparticles were intravenously injected into 4T1 tumor-bearing mice, and their NIR fluorescence and PA images were obtained. As shown in Figures 4A,B. AFSPN facilitated homologous targeting ability, and allowed to specifically target cancer-associated fibroblasts. The experiment results demonstrated that AF-SPN provided higher accumulation in tumor tissues than both the USPN and CC-SPN, and amplified NIR fluorescence and photoacoustic (PA) signals for tumor imaging (Li J. et al., 2018).

\section{HYBRID CELLS MEMBRANE CAMOUFLAGED THERANOSTIC NANOCOMPLEX}

Based on the concept that membranes from various cell types carry different properties. Research Scientists developed two types of cell membrane fusion coating made nanoparticles inherit and amplify the properties of both source cells (Liang et al., 2018; Ye H. et al., 2019). The two types of pre-extracted cell membranes were mixed together at appropriate protein weight ratios at $37^{\circ} \mathrm{C}$ to facilitate membrane fusion. For instance, Dehaini et al. fabricated RBC-platelet hybrid membrane-coated nanoparticles. This dual-membrane-coated nanoplatform exhibited long circulation, excellent biocompatibility and suitability for further in vivo exploration (Dehaini et al., 2017). In another study, firstly, the researchers get hybrid cells. Briefly, cancerous 4T1 cells and dendritic cells (DCs) were mixed at a ratio of 1:2 in the phosphate buffer (PBS) solution containing 50\% polyethylene glycol (PEG) $(M W=4000)$ and $10 \%$ dimethyl sulfoxide (DMSO) after $2 \mathrm{~min}$ fusion at $38^{\circ} \mathrm{C}$, the cells were washed with medium to remove the PEG and DMSO. After fused cells were cultured for 6-day, the cytomembranes (FMs) of hybrid cells were collected. FMs were coated on metal organic framework (PCN-224) by ultrasonic treatment in a cold water bath until the solution was transparent. The obtained hybrid cell membrane coated nanoparticles were further purified by centrifugation to remove the free FMs. The authors showed that this hybrid cell membrane coated nanoparticles can not only inherit the specifically targeted ability to homologous tumors from parent 4T1 cells but also obtained the enhanced ability of immune induction owing to the high 


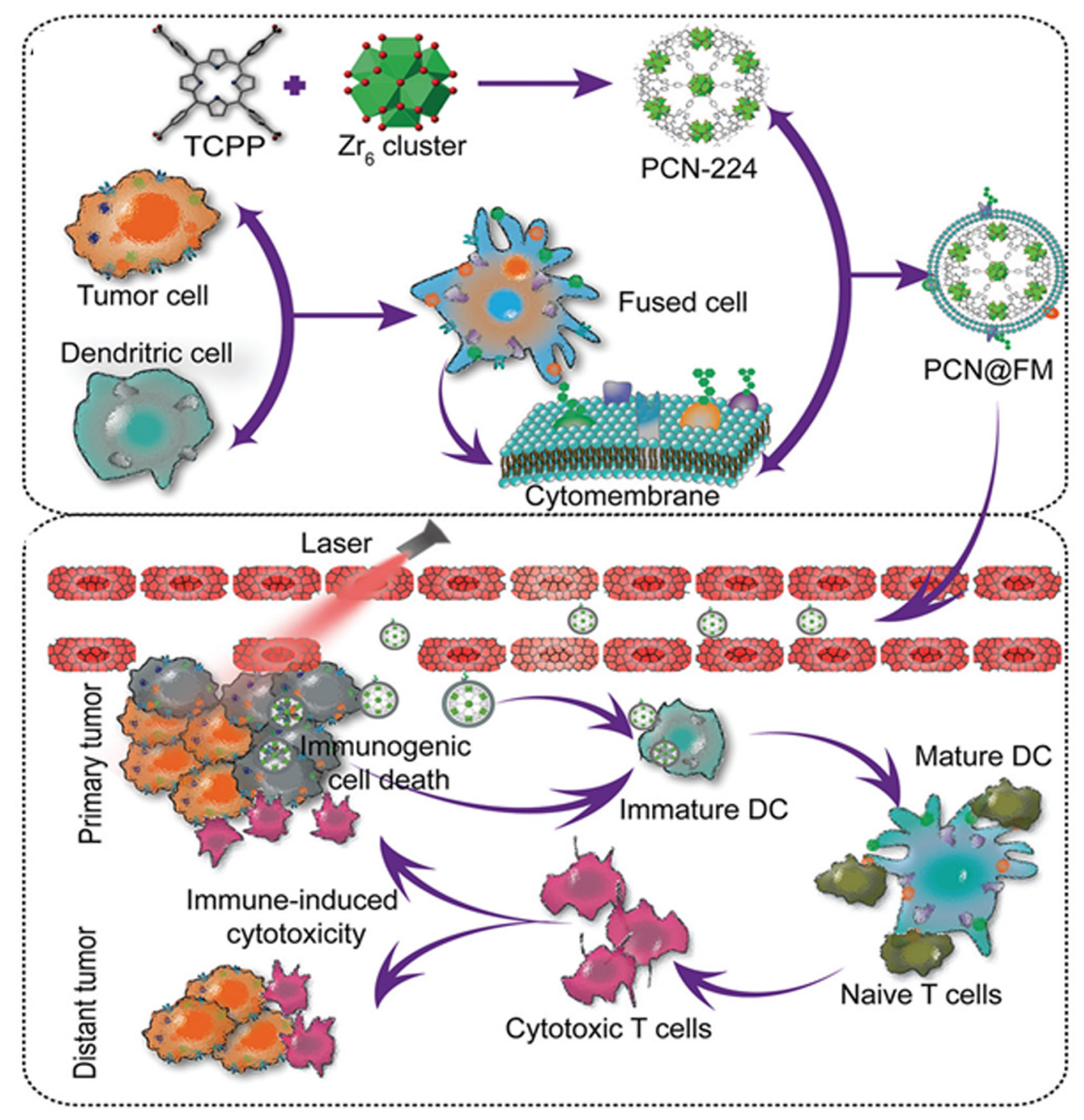

FIGURE 5 | Schematic illustration showed the preparation of PCN@FM for combined tumor therapy. Reproduced with permission from Liu W.L. et al. (2019). Copyright @ 2019 WILEY-VCH Verlag GmbH \& Co. KGaA, Weinheim.

expression of a whole array of tumor antigens in FMs (Figure 5; Liu W.L. et al., 2019).

\section{OTHER CELLS MEMBRANE CAMOUFLAGED THERANOSTIC NANOCOMPLEX}

Thanks to the advantages of cell membrane coated nanosystem for cancer theranostic, more and more types of cell membrane be used as coating materials according to their self-nature and the clinical need. Platelet, derived from megakaryocytes, is an indispensable component of blood stream, participate in many physiological activities and play an important role, including coagulation, hemostasis, the body's innate immune response, and cancer metastasis (Li Z. et al., 2018). P-Selectin is a cell adhesion protein, found predominantly in endothelial cells and platelets. Upon platelet activation, it can get exposed on the platelet membrane (PM) surface, and specifically bind to CD44 receptors upregulated on the surface of cancer cells (Bergstrand et al., 2019). Inspired by these properties of platelets, $\mathrm{Hu}$ et al. developed a PM coated core-shell nanovehicle (denoted as PM-NV) for codelivery of tumor necrosis factor (TNF)-related apoptosis inducing ligand (TRAIL) and doxorubicin (Dox). The nanocomplex was defined as TRAIL-Dox-PM-NV. TRAIL is one of the most important extracellular activators of apoptosis, induces apoptosis of tumor cells by binding to the death receptors (DR4, DR5) on the cell surface; while Dox can damage the nuclear DNA of cancer cells to trigger the intrinsic apoptosis signaling pathway. PM coating enhanced drug accumulation by active 
targeting based on the affinity between PM and overexpressed CD44 receptors on the cancer cells. TRAIL-Dox-PM-NV showed synergetic antitumor efficacy to MDA-MB-231 tumor-bearing nude mice (Hu et al., 2015).

At present, the great benefits of immunotherapies in oncology are evident, $\mathrm{T}$ cells engineered to express chimeric antigen receptors (CARs) that are specific for tumor antigens have demonstrated tremendous success in eradicating hematologic malignancies (e.g., CD19 CARs in leukemias) (Herzyk and Haggerty, 2018; Labanieh et al., 2018). However, this success was not observed in solid tumors, and the reasons for this are being investigated (Newick et al., 2017). Considering these situations, Ma et al. combined cell membrane coating nanotechnology with CAR-T therapy to treat hepatocellular carcinoma (HCC), due to the high tumor specificity of CAR-T cells and the advantage of cell membrane-camouflaged nanoparticles in drug delivery. They used Glypican-3 (GPC3) targeting CAR-T to prepare CAR-T membranes (CMs). GPC3, a 580-AA heparin sulfate proteoglycan, is a key biomarker for early diagnosis of HCC due to its overexpression in $75 \%$ of HCC samples, but not in healthy liver or other normal tissues (Dargel et al., 2015; Zhang Q. et al., 2018). Near-infrared (NIR) dye IR780, was loaded in mesoporous silica nanoparticles (MSNs) to form a core. The IR780 dye with NIR absorbance can produce heat under laser for PTT. IR780loaded MSNs (IMs) were coated with a layer of pre-prepared CAR-T membranes using an extrusion method to fabricate tumor specific CAR-T Cell membrane-coated nanoparticles (CIMs). CIMs inherited the tumor targeting and a long circulation ability from the membrane cloaking, and demonstrated enhanced antitumor capabilities with minimal systematic toxicity both in vitro and vivo (Ma et al., 2020b).

\section{CONCLUSION AND PERSPECTIVES}

This review has highlighted the current development of cell membrane coated nanoparticles for cancer theranostic. We present the overview of the application of RBC membrane coating materials, cancer cell membrane materials, stem cell membrane materials, and others on cancer theranostic. Cell membrane coated nanoparticles have shown unique advantages to enhance cancer therapy and imaging, but they still have

\section{REFERENCES}

Bergstrand, J., Xu, L., Miao, X., Li, N., Oktem, O., Franzen, B., et al. (2019). Superresolution microscopy can identify specific protein distribution patterns in platelets incubated with cancer cells. Nanoscale 11, 10023-10033. doi: 10.1039/ c9nr01967g

Bort, G., Lux, F., Dufort, S., Cremillieux, Y., Verry, C., and Tillement, O. (2020). EPR-mediated tumor targeting using ultrasmall-hybrid nanoparticles: from animal to human with theranostic AGuIX nanoparticles. Theranostics 10, 1319-1331. doi: 10.7150/thno.37543

Bose, R. J. C., Paulmurugan, R., Moon, J., Lee, S. H., and Park, H. (2018a). Cell membrane-coated nanocarriers: the emerging targeted delivery system for cancer theranostics. Drug Discov. Today 23, 891-899. doi: 10.1016/j.drudis. 2018.02.001

Bose, R. J. C., Uday Kumar, S., Zeng, Y., Afjei, R., Robinson, E., Lau, K., et al. (2018b). Tumor cell-derived extracellular vesicle-coated nanocarriers: an many problems need to be overcome in translating to the clinic. For example, the yield of cell membrane extraction is low, it often needs to culture a huge number of cells, and just harvest a small amount of cell membrane, therefore cell isolation and purification approach still requires future improvement. There are various proteins are present on the cell membrane. It also needs to identify the potential proteins and remove unwanted proteins. Although a large number of cell membrane coated nanoparticles have been developed for the integration of cancer diagnosis and treatment, how many of their specific functions have been developed, and whether they have realized the functions envisioned by researchers, are the urgent problems need to be proven. All in all, there is the need to establish standard protocols for obtaining and testing cell membrane coating production. However, the current evaluation of the therapeutic and diagnostic effect of cell membrane coated nanoparticles have been limited in preclinical studies. we hope that the clinical translation of cell membrane coated nanoparticles can be accelerated, which will make a positive impact on human health and be of great economic value.

\section{AUTHOR CONTRIBUTIONS}

TL contributed to the design, reorganize the figures, and writing the manuscript. XQ, YL, and XS contributed to research the literature. SL, HY, CW, CZ, JZ, and FY helped with editing the manuscript. YL conceived and designed the outline of this review. All the authors read and approved the final manuscript.

\section{FUNDING}

This work was supported, in part or in whole, by the National Natural Science Foundation of China (81671821, U19A2006, 11772088, 31700811, 11802056, 31800780, 11972111, and 31900940), the China Postdoctoral Science Foundation (2018M640904 and 2019T120831), the Sichuan Science and Technology Program (2019YJ0183 and 2019YJ0184), and the Fundamental Research Funds for the Central Universities (ZYGX2019J117).

efficient theranostic platform for the cancer-specific delivery of anti-miR-21 and imaging agents. ACS Nano 12, 10817-10832. doi: 10.1021/acsnano.8b02587

Cai, D., Liu, L., Han, C., Ma, X., Qian, J., Zhou, J., et al. (2019). Cancer cell membrane-coated mesoporous silica loaded with superparamagnetic ferroferric oxide and Paclitaxel for the combination of Chemo/Magnetocaloric therapy on MDA-MB-231 cells. Sci. Rep. 9:14475. doi: 10.1038/s41598-01951029-8

Chen, B., Dai, W., Mei, D., Liu, T., He, B., He, B., et al. (2016). Comprehensively priming the tumor microenvironment by cancer-associated fibroblast-targeted liposomes for combined therapy with cancer cell-targeted chemotherapeutic drug delivery system. J. Control. Release 241, 68-80. doi: 10.1016/j.jconrel.2016. 09.014

Chen, Z., Wang, Z., and Gu, Z. (2019). Bioinspired and biomimetic nanomedicines. Acc Chem. Res. 52, 1255-1264. doi: 10.1021/acs.accounts.9b00079

Chen, Z., Zhao, P., Luo, Z., Zheng, M., Tian, H., Gong, P., et al. (2016). Cancer cell membrane-biomimetic nanoparticles for homologous-targeting dual-modal 
imaging and photothermal therapy. ACS Nano 10, 10049-10057. doi: 10.1021/ acsnano.6b04695

Choi, K. Y., Liu, G., Lee, S., and Chen, X. (2012). Theranostic nanoplatforms for simultaneous cancer imaging and therapy: current approaches and future perspectives. Nanoscale 4, 330-342. doi: 10.1039/c1nr11277e

Dargel, C., Bassani-Sternberg, M., Hasreiter, J., Zani, F., Bockmann, J. H., Thiele, F., et al. (2015). T cells engineered to express a T-cell receptor specific for Glypican3 to recognize and kill hepatoma cells in vitro and in mice. Gastroenterology 149 , 1042-1052. doi: 10.1053/j.gastro.2015.05.055

Dehaini, D., Wei, X., Fang, R. H., Masson, S., Angsantikul, P., Luk, B. T., et al. (2017). Erythrocyte-platelet hybrid membrane coating for enhanced nanoparticle functionalization. Adv. Mater. 29:10.1002/adma.201606209. doi: 10.1002/adma.201606209

Evangelopoulos, M., and Tasciotti, E. (2017). Bioinspired approaches for cancer nanotheranostics. Nanomedicine 12, 5-7. doi: 10.2217/nnm-2016-0374

Fang, R. H., Kroll, A. V., Gao, W., and Zhang, L. (2018). Cell membrane coating nanotechnology. Adv. Mater. 30:e1706759. doi: 10.1002/adma.201706759

Feng, Q., Yang, X., Hao, Y., Wang, N., Feng, X., Hou, L., et al. (2019). Cancer cell membrane-biomimetic nanoplatform for enhanced sonodynamic therapy on breast cancer via autophagy regulation strategy. ACS Appl. Mater. Interfaces. 11, 32729-32738. doi: 10.1021/acsami.9b10948

Gao, C., Lin, Z., Jurado-Sanchez, B., Lin, X., Wu, Z., and He, Q. (2016). Stem cell membrane-coated nanogels for highly efficient in vivo tumor targeted drug delivery. Small 12, 4056-4062. doi: 10.1002/smll.201600624

Harris, J. C., Scully, M. A., and Day, E. S. (2019). Cancer cell membrane-coated nanoparticles for cancer management. Cancers 11:10.1002/adma.201606209. doi: $10.3390 /$ cancers 11121836

Herzyk, D. J., and Haggerty, H. G. (2018). Cancer immunotherapy: factors important for the evaluation of safety in nonclinical studies. AAPS J. 20:28. doi: $10.1208 /$ s12248-017-0184-3

Hsieh, C. C., Kang, S. T., Lin, Y. H., Ho, Y. J., Wang, C. H., Yeh, C. K., et al. (2015). Biomimetic acoustically-responsive vesicles for theranostic applications. Theranostics 5, 1264-1274. doi: 10.7150/thno.11848

Hu, C. M. J., Zhang, L., Aryal, S., Cheung, C., Fang, R. H., and Zhang, L. F. (2011). Erythrocyte membrane-camouflaged polymeric nanoparticles as a biomimetic delivery platform. Proc. Natl. Acad. Sci. U.S.A. 108, 10980-10985. doi: 10.1073/ pnas. 1106634108

Hu, Q., Sun, W., Qian, C., Wang, C., Bomba, H. N., and Gu, Z. (2015). Anticancer platelet-mimicking nanovehicles. Adv. Mater. 27, 7043-7050. doi: 10.1002/ adma.201503323

Ji, T., Ding, Y., Zhao, Y., Wang, J., Qin, H., Liu, X., et al. (2015). Peptide assembly integration of fibroblast-targeting and cell-penetration features for enhanced antitumor drug delivery. Adv. Mater. 27, 1865-1873. doi: 10.1002/ adma.201404715

Ji, T., Zhao, Y., Ding, Y., Wang, J., Zhao, R., Lang, J., et al. (2016). Transformable peptide nanocarriers for expeditious drug release and effective cancer therapy via cancer-associated fibroblast activation. Angew. Chem. Int. Ed. Engl. 55, 1050-1055. doi: 10.1002/anie.201506262

Jiang, Q., Luo, Z., Men, Y., Yang, P., Peng, H., Guo, R., et al. (2017). Red blood cell membrane-camouflaged melanin nanoparticles for enhanced photothermal therapy. Biomaterials 143, 29-45. doi: 10.1016/j.biomaterials.2017.07.027

Jin, J., Krishnamachary, B., Barnett, J. D., Chatterjee, S., Chang, D., Mironchik, Y., et al. (2019). Human cancer cell Membrane-coated biomimetic nanoparticles reduce fibroblast-mediated invasion and metastasis and induce T-Cells. ACS Appl. Mater. Interfaces 11, 7850-7861. doi: 10.1021/acsami.8b22309

Jing, X., Xu, Y., Liu, D., Wu, Y., Zhou, N., Wang, D., et al. (2019). Intelligent nanoflowers: a full tumor microenvironment-responsive multimodal cancer theranostic nanoplatform. Nanoscale 11, 15508-15518. doi: 10.1039/ c9nr04768a

Kovacs, D., Igaz, N., Marton, A., Ronavari, A., Belteky, P., Bodai, L., et al. (2020). Core-shell nanoparticles suppress metastasis and modify the tumoursupportive activity of cancer-associated fibroblasts. J. Nanobiotechnol. 18:18. doi: 10.1186/s12951-020-0576-x

Kroll, A. V., Fang, R. H., and Zhang, L. (2017). Biointerfacing and applications of cell membrane-coated nanoparticles. Bioconjug. Chem. 28, 23-32. doi: 10.1021/ acs.bioconjchem.6b00569

Kumar, P., Treuren, T. V., Ranjan, A. P., Chaudhary, P., and Vishwanatha, J. K. (2019). In vivo imaging and biodistribution of near infrared dye loaded brain-metastatic-breast-cancer-cell-membrane coated polymeric nanoparticles. Nanotechnology 30:265101. doi: 10.1088/1361-6528/ab0f46

Labanieh, L., Majzner, R. G., and Mackall, C. L. (2018). Programming CAR-T cells to kill cancer. Nat. Biomed. Eng. 2, 377-391. doi: 10.1038/s41551-018$0235-9$

Letko Khait, N., Malkah, N., Kaneti, G., Fried, L., Cohen Anavy, N., Bronshtein, T., et al. (2019). Radiolabeling of cell membrane-based nano-vesicles with (14)Clinoleic acid for robust and sensitive quantification of their biodistribution. J. Control. Release 293, 215-223. doi: 10.1016/j.jconrel.2018.12.005

Li, C., Yang, X. Q., An, J., Cheng, K., Hou, X. L., Zhang, X. S., et al. (2020). Red blood cell membrane-enveloped $\mathrm{O}_{2}$ self-supplementing biomimetic nanoparticles for tumor imaging-guided enhanced sonodynamic therapy. Theranostics 10 , 867-879. doi: 10.7150/thno.37930

Li, J., Zhen, X., Lyu, Y., Jiang, Y., Huang, J., and Pu, K. (2018). Cell membrane coated semiconducting polymer nanoparticles for enhanced multimodal cancer phototheranostics. ACS Nano 12, 8520-8530. doi: 10.1021/acsnano.8b04066

Li, L., Zhou, S., Lv, N., Zhen, Z., Liu, T., Gao, S., et al. (2018). Photosensitizerencapsulated ferritins mediate photodynamic therapy against cancer-associated fibroblasts and improve tumor accumulation of nanoparticles. Mol. Pharm. 15, 3595-3599. doi: 10.1021/acs.molpharmaceut.8b00419

Li, S. Y., Xie, B. R., Cheng, H., Li, C. X., Zhang, M. K., Qiu, W. X., et al. (2018). A biomimetic theranostic $\mathrm{O}_{2}$-meter for cancer targeted photodynamic therapy and phosphorescence imaging. Biomaterials 151, 1-12. doi: 10.1016/j. biomaterials.2017.10.021

Li, T., Shen, X., Geng, Y., Chen, Z., Li, L., Li, S., et al. (2016). Folate-functionalized magnetic-mesoporous silica nanoparticles for drug/gene codelivery to potentiate the antitumor efficacy. ACS Appl. Mater. Interfaces 8, 13748-13758. doi: $10.1021 /$ acsami.6b02963

Li, T., Shi, S., Goel, S., Shen, X., Xie, X., Chen, Z., et al. (2019). Recent advancements in mesoporous silica nanoparticles towards therapeutic applications for cancer. Acta Biomater. 89, 1-13. doi: 10.1016/j.actbio.2019.02.031

Li, W., Yang, J., Luo, L., Jiang, M., Qin, B., Yin, H., et al. (2019). Targeting photodynamic and photothermal therapy to the endoplasmic reticulum enhances immunogenic cancer cell death. Nat. Commun. 10:3349. doi: 10.1038/ s41467-019-11269-8

$\mathrm{Li}, \mathrm{Z} ., \mathrm{Hu}, \mathrm{S}$., and Cheng, K. (2018). Platelets and their biomimetics for regenerative medicine and cancer therapies. J. Mater. Chem. B 6, 7354-7365. doi: 10.1039/ C8TB02301H

Lian, Y., Wang, X., Guo, P., Li, Y., Raza, F., Su, J., et al. (2019). Erythrocyte membrane-coated arsenic trioxide-loaded sodium alginate nanoparticles for tumor therapy. Pharmaceutics 12:21. doi: 10.3390/pharmaceutics12010021

Liang, H., Huang, K., Su, T., Li, Z., Hu, S., Dinh, P. U., et al. (2018). Mesenchymal stem cell/red blood cell-inspired nanoparticle therapy in mice with carbon tetrachloride-induced acute liver failure. ACS Nano 12, 6536-6544. doi: 10. $1021 /$ acsnano.8b00553

Liu, C., Wang, D., Zhang, S., Cheng, Y., Yang, F., Xing, Y., et al. (2019). Biodegradable biomimic copper/manganese silicate nanospheres for chemodynamic/photodynamic synergistic therapy with simultaneous glutathione depletion and hypoxia relief. ACS Nano 13, 4267-4277. doi: 10. 1021/acsnano.8b09387

Liu, S., Wang, L., Zhang, M., Tao, K., Wang, B., Lin, M., et al. (2019). Tumor microenvironment-responsive nanoshuttles with sodium citrate modification for hierarchical targeting and improved tumor theranostics. ACS Appl. Mater. Interfaces 11, 25730-25739. doi: 10.1021/acsami.9b07957

Liu, J. M., Zhang, D. D., Fang, G. Z., and Wang, S. (2018). Erythrocyte membrane bioinspired near-infrared persistent luminescence nanocarriers for in vivo longcirculating bioimaging and drug delivery. Biomaterials 165, 39-47. doi: 10.1016/ j.biomaterials.2018.02.042

Liu, W. L., Zou, M. Z., Liu, T., Zeng, J. Y., Li, X., Yu, W. Y., et al. (2019). Expandable immunotherapeutic nanoplatforms engineered from cytomembranes of hybrid cells derived from cancer and dendritic cells. Adv. Mater. 31:e1900499. doi: 10.1002/adma.201900499

Liu, X., Su, H., Shi, W., Liu, Y., Sun, Y., and Ge, D. (2018). Functionalized poly(pyrrole-3-carboxylic acid) nanoneedles for dual-imaging guided PDT/PTT combination therapy. Biomaterials 167, 177-190. doi: 10.1016/j.biomaterials.2018.03.030

Liu, Y., Zhen, W., Jin, L., Zhang, S., Sun, G., Zhang, T., et al. (2018). All-in-one theranostic nanoagent with enhanced reactive oxygen species generation and 
modulating tumor microenvironment ability for effective tumor eradication. ACS Nano 12, 4886-4893. doi: 10.1021/acsnano.8b01893

Ma, J., Zhang, S., Liu, J., Liu, F., Du, F., Li, M., et al. (2019). Targeted drug delivery to stroke via chemotactic recruitment of nanoparticles coated with membrane of engineered neural stem cells. Small 15:e1902011. doi: 10.1002/smll.201902011

Ma, W., Sha, S. N., Chen, P. L., Yu, M., Chen, J. J., Huang, C. B., et al. (2020a). A cell membrane-targeting self-delivery chimeric peptide for enhanced photodynamic therapy and in situ therapeutic feedback. Adv. Healthc Mater. 9:e1901100. doi: 10.1002/adhm.201901100

Ma, W., Zhu, D., Li, J., Chen, X., Xie, W., Jiang, X., et al. (2020b). Coating biomimetic nanoparticles with chimeric antigen receptor $\mathrm{T}$ cell-membrane provides high specificity for hepatocellular carcinoma photothermal therapy treatment. Theranostics 10, 1281-1295. doi: 10.7150/thno.40291

Madamsetty, V. S., Mukherjee, A., and Mukherjee, S. (2019). Recent trends of the bio-inspired nanoparticles in cancer theranostics. Front. Pharmacol. 10:1264. doi: 10.3389/fphar.2019.01264

Meng, Q.-F., Cheng, Y.-X., Huang, Q., Zan, M., Xie, W., Sun, Y., et al. (2019). Biomimetic immunomagnetic nanoparticles with minimal nonspecific biomolecule adsorption for enhanced isolation of circulating tumor cells. ACS Appl. Mater. Interfaces 11, 28732-28739. doi: 10.1021/acsami.9b10318

Meng, Q. F., Rao, L., Zan, M., Chen, M., Yu, G. T., Wei, X., et al. (2018). Macrophage membrane-coated iron oxide nanoparticles for enhanced photothermal tumor therapy. Nanotechnology 29:134004. doi: 10.1088/13616528/aaa7c7

Min, H., Wang, J., Qi, Y., Zhang, Y., Han, X., Xu, Y., et al. (2019). Biomimetic metal-organic framework nanoparticles for cooperative combination of antiangiogenesis and photodynamic therapy for enhanced efficacy. Adv. Mater. 31:e1808200. doi: 10.1002/adma.201808200

Narain, A., Asawa, S., Chhabria, V., and Patil-Sen, Y. (2017). Cell membrane coated nanoparticles: next-generation therapeutics. Nanomedicine 12, 2677-2692. doi: 10.2217/nnm-2017-0225

Newick, K., O’Brien, S., Moon, E., and Albelda, S. M. (2017). CAR T cell therapy for solid tumors. Annu. Rev. Med. 68, 139-152. doi: 10.1146/annurev-med062315-120245

Nie, D., Dai, Z., Li, J., Yang, Y., Xi, Z., Wang, J., et al. (2019). Cancer-cellmembrane-coated nanoparticles with a yolk-shell structure augment cancer chemotherapy. Nano Lett. 20, 936-946. doi: 10.1021/acs.nanolett.9b03817

Oldenborg, P. A., Zheleznyak, A., Fang, Y. F., Lagenaur, C. F., Gresham, H. D., and Lindberg, F. P. (2000). Role of CD47 as a marker of self on red blood cells. Science 288, 2051-2054.

Pasto, A., Giordano, F., Evangelopoulos, M., Amadori, A., and Tasciotti, E. (2019). Cell membrane protein functionalization of nanoparticles as a new tumortargeting strategy. Clin. Transl. Med. 8:8. doi: 10.1186/s40169-019-0224-y

Phillips, E., Penate-Medina, O., Zanzonico, P. B., Carvajal, R. D., Mohan, P., Ye, Y., et al. (2014). Clinical translation of an ultrasmall inorganic optical-PET imaging nanoparticle probe. Sci. Transl. Med. 6:260ra149. doi: 10.1126/scitranslmed. 3009524

Rao, L., Meng, Q.-F., Bu, L.-L., Cai, B., Huang, Q., Sun, Z.-J., et al. (2017). Erythrocyte membrane-coated upconversion nanoparticles with minimal protein adsorption for enhanced tumor imaging. ACS Appl. Mater. Interfaces 9, 2159-2168. doi: 10.1021/acsami.6b14450

Ray, S., Li, Z., Hsu, C. H., Hwang, L. P., Lin, Y. C., Chou, P. T., et al. (2018). Dendrimer- and copolymer-based nanoparticles for magnetic resonance cancer theranostics. Theranostics 8, 6322-6349. doi: 10.7150/thno.27828

Ren, H., Liu, J., Li, Y., Wang, H., Ge, S., Yuan, A., et al. (2017). Oxygen self-enriched nanoparticles functionalized with erythrocyte membranes for long circulation and enhanced phototherapy. Acta Biomater. 59, 269-282. doi: 10.1016/j.actbio. 2017.06.035

Roma-Rodrigues, C., Pombo, I., Raposo, L., Pedrosa, P., Fernandes, A. R., and Baptista, P. V. (2019). Nanotheranostics targeting the tumor microenvironment. Front. Bioeng. Biotechnol. 7:197. doi: 10.3389/fbioe. 2019.00197

Shao, C., Xiao, F., Guo, H., Yu, J., Jin, D., Wu, C., et al. (2019). Utilizing polymer micelle to control dye J-aggregation and enhance its theranostic capability. iScience 22, 229-239. doi: 10.1016/j.isci.2019.11.022

Shao, D., Li, M., Wang, Z., Zheng, X., Lao, Y. H., Chang, Z., et al. (2018). Bioinspired diselenide-bridged mesoporous silica nanoparticles for dual-responsive protein delivery. Adv. Mater. e1801198. doi: 10.1002/adma. 201801198 Epub ahead of print].

Shen, X., Li, T., Chen, Z., Xie, X., Zhang, H., Feng, Y., et al. (2019). NIR-lighttriggered anticancer strategy for dual-modality imaging-guided combination therapy via a bioinspired hybrid PLGA nanoplatform. Mol. Pharm. 16, 13671384. doi: 10.1021/acs.molpharmaceut.8b01321

Shin, T. H., Lee, D. Y., Ketebo, A. A., Lee, S., Manavalan, B., Basith, S., et al. (2019). Silica-coated magnetic nanoparticles decrease human bone marrow-derived mesenchymal stem cell migratory activity by reducing membrane fluidity and impairing focal adhesion. Nanomaterials 9:1475. doi: 10.3390/nano9101475

Su, J., Sun, H., Meng, Q., Zhang, P., Yin, Q., and Li, Y. (2017). Enhanced blood suspensibility and laser-activated tumor-specific drug release of theranostic mesoporous silica nanoparticles by functionalizing with erythrocyte membranes. Theranostics 7, 523-537. doi: 10.7150/thno.17259

Sung, S. Y., Su, Y. L., Cheng, W., Hu, P. F., Chiang, C. S., Chen, W. T., et al. (2019). Graphene quantum dots-mediated theranostic penetrative delivery of drug and photolytics in deep tumors by targeted biomimetic nanosponges. Nano Lett. 19, 69-81. doi: 10.1021/acs.nanolett.8b03249

Vankayala, R., and Hwang, K. C. (2018). Near-infrared-light-activatable nanomaterial-mediated phototheranostic nanomedicines: an emerging paradigm for cancer treatment. Adv. Mater. 30:e1706320. doi: 10.1002/adma. 201706320

Wang, J., Wang, Z., Zhong, Y., Zou, Y., Wang, C., Wu, H., et al. (2020). Central metal-derived co-assembly of biomimetic GdTPP/ZnTPP porphyrin nanocomposites for enhanced dual-modal imaging-guided photodynamic therapy. Biomaterials 229:119576. doi: 10.1016/j.biomaterials.2019.119576

Wang, P., Wang, X., Luo, Q., Li, Y., Lin, X., Fan, L., et al. (2019). Fabrication of red blood cell-based multimodal theranostic probes for second near-infrared window fluorescence imaging-guided tumor surgery and photodynamic therapy. Theranostics 9, 369-380. doi: 10.7150/thno.29817

Wu, C., Tian, Y., Zhang, Y., Xu, J., Wang, Y., Guan, X., et al. (2020). Acid-triggered charge-convertible graphene-based all-in-one nanocomplex for enhanced genetic phototherapy of triple-negative breast cancer. Adv. Healthc. Mater. 9:e1901187. doi: 10.1002/adhm.201901187

Wu, M., Le, W., Mei, T., Wang, Y., Chen, B., Liu, Z., et al. (2019). Cell membrane camouflaged nanoparticles: a new biomimetic platform for cancer photothermal therapy. Int. J. Nanomed. 14, 4431-4448. doi: 10.2147/IJN.S200284

Xie, J., Shen, Q., Huang, K., Zheng, T., Cheng, L., Zhang, Z., et al. (2019). Oriented assembly of cell-mimicking nanoparticles via a molecular affinity strategy for targeted drug delivery. ACS Nano 13, 5268-5277. doi: 10.1021/ acsnano.8b09681

Xie, X., Chen, Y., Chen, Z., Feng, Y., Wang, J., Li, T., et al. (2019). Polymeric hybrid nanomicelles for cancer theranostics: an efficient and precise anticancer strategy for the codelivery of doxorubicin/miR-34a and magnetic resonance imaging. ACS Appl. Mater. Interfaces 11, 43865-43878. doi: 10.1021/acsami.9b14908

Xu, C., Nam, J., Hong, H., Xu, Y., and Moon, J. J. (2019). Positron emission tomography-guided photodynamic therapy with biodegradable mesoporous silica nanoparticles for personalized cancer immunotherapy. ACS Nano 13, 12148-12161. doi: 10.1021/acsnano.9b06691

Yan, H., Shao, D., Lao, Y. H., Li, M., Hu, H., and Leong, K. W. (2019). Engineering cell membrane-based nanotherapeutics to target inflammation. Adv. Sci. 6:1900605. doi: 10.1002/advs.201900605

Yang, H., Chen, Y., Chen, Z., Geng, Y., Xie, X., Shen, X., et al. (2017). Chemo-photodynamic combined gene therapy and dual-modal cancer imaging achieved by $\mathrm{pH}$-responsive alginate/chitosan multilayer-modified magnetic mesoporous silica nanocomposites. Biomater. Sci. 5, 1001-1013. doi: 10.1039/ c7bm00043j

Yang, H., Shen, X., Yan, J., Xie, X., Chen, Z., Li, T., et al. (2018). Chargereversal-functionalized PLGA nanobubbles as theranostic agents for ultrasonicimaging-guided combination therapy. Biomater. Sci. 6, 2426-2439. doi: 10. 1039/c8bm00419f

Yang, J., Lu, W., Xiao, J., Zong, Q., Xu, H., Yin, Y., et al. (2018). A positron emission tomography image-guidable unimolecular micelle nanoplatform for cancer theranostic applications. Acta Biomater. 79, 306-316. doi: 10.1016/j. actbio.2018.08.036

Yang, N., Ding, Y., Zhang, Y., Wang, B., Zhao, X., Cheng, K., et al. (2018). Surface functionalization of polymeric nanoparticles with umbilical cord-derived 
mesenchymal stem cell membrane for tumor-targeted therapy. ACS Appl. Mater. Interfaces 10, 22963-22973. doi: 10.1021/acsami.8b05363

Yang, Q., Xiao, Y., Yin, Y., Li, G., and Peng, J. (2019). Erythrocyte membranecamouflaged IR780 and DTX coloading polymeric nanoparticles for imagingguided cancer photo-chemo combination therapy. Mol. Pharm. 16, 3208-3220. doi: 10.1021/acs.molpharmaceut.9b00413

Ye, D., Shuhendler, A. J., Cui, L., Tong, L., Tee, S. S., Tikhomirov, G., et al. (2014). Bioorthogonal cyclization-mediated in situ self-assembly of small-molecule probes for imaging caspase activity in vivo. Nat. Chem. 6, 519-526. doi: 10.1038/ nchem. 1920

Ye, H., Wang, K., Wang, M., Liu, R., Song, H., Li, N., et al. (2019). Bioinspired nanoplatelets for chemo-photothermal therapy of breast cancer metastasis inhibition. Biomaterials 206, 1-12. doi: 10.1016/j.biomaterials.2019.03.024

Ye, S., Wang, F., Fan, Z., Zhu, Q., Tian, H., Zhang, Y., et al. (2019). Light/pHtriggered biomimetic red blood cell membranes camouflaged small molecular drug assemblies for imaging-guided combinational chemo-photothermal therapy. ACS Appl. Mater. Interfaces 11, 15262-15275. doi: 10.1021/acsami. $9 \mathrm{~b} 00897$

Yue, W., Chen, L., Yu, L., Zhou, B., Yin, H., Ren, W., et al. (2019). Checkpoint blockade and nanosonosensitizer-augmented noninvasive sonodynamic therapy combination reduces tumour growth and metastases in mice. Nat. Commun. 10:2025. doi: 10.1038/s41467-019-09760-3

Zhang, D., Ye, Z., Wei, L., Luo, H., and Xiao, L. (2019). Cell membrane-coated porphyrin metal-organic frameworks for cancer cell targeting and $\mathrm{O}_{2}$-evolving photodynamic therapy. ACS Appl. Mater. Interfaces 11, 39594-39602. doi: 10. 1021/acsami.9b14084

Zhang, W., Yu, M., Xi, Z., Nie, D., Dai, Z., Wang, J., et al. (2019). Cancer cell membrane-camouflaged nanorods with endoplasmic reticulum targeting for improved antitumor therapy. ACS Appl. Mater. Interfaces 11, 46614-46625. doi: 10.1021/acsami.9b18388
Zhang, N., Li, M., Sun, X., Jia, H., and Liu, W. (2018). NIR-responsive cancer cytomembrane-cloaked carrier-free nanosystems for highly efficient and self-targeted tumor drug delivery. Biomaterials 159, 25-36. doi: 10.1016/ j.biomaterials.2018.01.007

Zhang, Q., Han, Z., Tao, J., Zhao, M., Zhang, W., Li, P., et al. (2018). An innovative peptide with high affinity to GPC3 for hepatocellular carcinoma diagnosis. Biomater. Sci. 7, 159-167. doi: 10.1039/c8bm01016a

Zhao, Q., Hai, B., Zhang, X., Xu, J., Koehler, B., and Liu, F. (2019). Biomimetic nanovesicles made from iPS cell-derived mesenchymal stem cells for targeted therapy of triple-negative breast cancer. Nanomedicine 24:102146. doi: 10.1016/ j.nano.2019.102146

Zhou, J., Kroll, A. V., Holay, M., Fang, R. H., and Zhang, L. (2019). Biomimetic nanotechnology toward personalized vaccines. Adv. Mater. 32:e1901255. doi: 10.1002/adma.201901255

Zhu, J. Y., Zheng, D. W., Zhang, M. K., Yu, W. Y., Qiu, W. X., Hu, J. J., et al. (2016). Preferential cancer cell self-recognition and tumor self-targeting by coating nanoparticles with homotypic cancer cell membranes. Nano Lett. 16, 5895-5901. doi: 10.1021/acs.nanolett.6b02786

Conflict of Interest: The authors declare that the research was conducted in the absence of any commercial or financial relationships that could be construed as a potential conflict of interest.

Copyright (C) $2020 \mathrm{Li}$, Qin, Li, Shen, Li, Yang, Wu, Zheng, Zhu, You and Liu. This is an open-access article distributed under the terms of the Creative Commons Attribution License (CC BY). The use, distribution or reproduction in other forums is permitted, provided the original author(s) and the copyright owner(s) are credited and that the original publication in this journal is cited, in accordance with accepted academic practice. No use, distribution or reproduction is permitted which does not comply with these terms. 